\title{
Higher mitochondrial DNA copy number is associated with lower prevalence of microalbuminuria
}

\author{
Jung Eun Lee ${ }^{1 *}$, Hansoo Park ${ }^{2 *}$, Young Seok Ju ${ }^{2,3}$, \\ Minhye Kwak ${ }^{2,3}$,Jong-II Kim ${ }^{2,4}$, Ha Young Oh ${ }^{1}$ \\ and Jeong-Sun $\mathrm{SeO}^{2,3,5,6}$ \\ ${ }^{1}$ Division of Nephrology \\ Samsung Medical Center \\ Sungkyunkwan University School of Medicine \\ Seoul 135-710, Korea \\ ${ }^{2}$ ILCHUN Genomic Medicine Institute \\ Medical Research Center \\ Seoul National University \\ Seoul 110-799, Korea \\ ${ }^{3}$ Department of Biochemistry and Molecular Biology \\ Seoul National University College of Medicine \\ Seoul 110-799, Korea \\ ${ }^{4}$ PSOMA Therapeutics Inc \\ Seoul 153-023, Korea \\ ${ }^{5}$ Macrogen Inc., Seoul 153-023, Korea \\ ${ }^{6}$ Corresponding author: Tel, 82-2-740-8246; \\ Fax, 82-2-741-5423; E-mail, jeongsun@snu.ac.kr \\ *These authors contributed equally to this work. \\ DOI 10.3858/emm.2009.41.4.028
}

\section{Accepted 26 November 2008}

Abbreviations: ACR, albumin to creatinine ratio; BMI, body mass index; $\mathrm{Cl}$, confidence interval; CKD, chronic kidney disease; CRP, C-reactive protein; GENDISCAN, GENe DIScovery for Complex traits in Asian population of Northeast; GFR, glomerular filtration rate; HOMA-IR, Homeostasis Model Assessment for insulin resistance; IRB, institutional review board; MDRD, Modification of Diet in Renal Disease; OR, odds ratio; ROS, reactive oxygen species; SBP, systolic blood pressure; WBC, white blood cell

\footnotetext{
Abstract

It has been suggested that mitochondrial dysfunction contributes to the initiation and development of atherosclerosis and cardiovascular disease. We examined the association between mitochondrial DNA (mtDNA) copy number and microalbuminuria in a cross-sectional community-based study. We measured peripheral blood mtDNA copy number in 694 adults without chronic kidney disease by a real-time PCR method. The overall prevalence of microalbuminuria (defined as an albumin creatinine ratio of 30 to $299 \mathrm{mg} / \mathrm{g}$ ) was $4.5 \%$. The prevalence of microalbuminuria decreased progressively from the lower to the upper quartiles of
}

mtDNA copy number $(6.9 \%, 5.7 \%, 2.9 \%$, and $2.3 \%$ in quartiles $1,2,3$, and 4, respectively, $P=0.017$ for trend). Multiple logistic regression models showed that the quartile of mtDNA copy number was independently associated with the prevalence of microalbuminuria $(P=$ 0.01 for trend). Compared with the lowest quartile, the highest quartile had an odds ratio of 0.22 for microalbuminuria ( $95 \%$ confidence interval, 0.05 to $0.87 ; P=$ $0.03)$. Higher mtDNA copy number was associated with the lower prevalence of microalbuminuria in a community-based population.

Keywords: albuminuria; cardiovascular diseases; DNA, mitochondrial; mitochondria; oxidative phosphorylation

\section{Introduction}

The mitochondrion is the major cellular site of energy production and respiration involving the process of oxidative phosphorylation. Mitochondrial defects have been implicated in the pathogenesis of insulin resistance and type 2 diabetes (Lee et al., 1998; Song et al., 2001; Petersen et al., 2003, 2004; Lowell and Shulman, 2005). Several lines of evidence suggest that mitochondrial dysfunction contributes to the initiation and development of atherosclerosis and cardiovascular disease (CorralDebrinski et al., 1992; Ballinger et al., 2002; KnightLozano et al., 2002; Madamanchi and Runge, 2007; Nisoli et al., 2007). Mitochondrial DNA (mtDNA) is a $16.5 \mathrm{~kb}$, double-stranded, closed circular molecule encoding 37 genes: 22 tRNAs, 2 rRNAs and 13 proteins, which is vital for maintaining normal mitochondrial function. Decreased mitochondrial activity might be caused, at least partly, by reduced content of mtDNA. Expression of nuclear-encoded genes involved in oxidative phosphorylation is downregulated in insulin-resistant skeletal muscle (Mootha et al., 2003). In addition, mtDNA copy number decreases by up to $50 \%$ in skeletal muscle of subjects with type 2 diabetes and is low in peripheral blood of offspring of those with type 2 diabetes when compared with matched controls without a family history (Antonetti et al., 1995). Thus, some investigators have speculated that mtDNA copy number might be a surrogate marker of mitochondrial function (Cho et al., 2007). Indeed, mtDNA copy number is closely associated with 
several cardiovascular risk factors including insulin resistance, aging, and abdominal obesity (Lee et al., 1998; Cho et al., 2007). However, few clinical studies have focused on the association between target organ damage and mtDNA copy number.

Microalbuminuria is associated with an increased risk of cardiovascular morbidity in patients with diabetes and hypertension, and in the general population. The amount of urinary albumin excretion is considered to reflect generalized endothelial dysfunction associated with a variety of risk factors (de Zeeuw, 2004).
Therefore, we investigated the relationship between urinary albumin excretion and mtDNA copy number in the community-based, cross-sectional study.

\section{Results}

Mean age of study population was $37.4 \pm 13.7$ years and $54.8 \%$ were women. The prevalence of hypertension was $12.3 \%$, diabetes was $2.9 \%$, and microalbuminuria was $4.5 \%$. Table 1 presents ba-

Table 1. Baseline characteristics of the study population by quartiles of mitochondrial DNA copy number.

\begin{tabular}{|c|c|c|c|c|c|}
\hline \multirow{2}{*}{ Variables } & \multicolumn{4}{|c|}{ Quartile of mitochondrial DNA copy number } & \multirow{2}{*}{$P$} \\
\hline & $\mathrm{I}(\leq 310)$ & II $(310-560)$ & III (560-912) & $\operatorname{IV}(>912)$ & \\
\hline Age $(y r)$ & $39 \pm 14$ & $38 \pm 14$ & $37 \pm 13$ & $35 \pm 13$ & 0.042 \\
\hline Women (\%) & $53.8 \%$ & $54.0 \%$ & $52.9 \%$ & $58.4 \%$ & NS \\
\hline Current smoker (\%) & $26.2 \%$ & $27.6 \%$ & $25.0 \%$ & $23.3 \%$ & NS \\
\hline Physical activity (\%) & $62.2 \%$ & $60.3 \%$ & $56.1 \%$ & $62.2 \%$ & NS \\
\hline $\mathrm{SBP}(\mathrm{mmHg})$ & $121 \pm 20$ & $120 \pm 18$ & $120 \pm 16$ & $120 \pm 16$ & NS \\
\hline BMI $\left(\mathrm{kg} / \mathrm{m}^{2}\right)$ & $25.5 \pm 4.8$ & $24.9 \pm 4.9$ & $25.0 \pm 4.0$ & $25.3 \pm 4.4$ & NS \\
\hline Waist circumference $(\mathrm{cm})$ & $84.0 \pm 12.7$ & $81.7 \pm 12.2$ & $82.5 \pm 10.7$ & $82.2 \pm 11.0$ & NS \\
\hline Glucose (mg/dl) & $98 \pm 32$ & $96 \pm 15$ & $96 \pm 15$ & $94 \pm 13$ & NS \\
\hline HOMA-IR score & $1.73(1.33-2.37)$ & $1.66(1.15-2.27)$ & $1.80(1.16-2.41)$ & $1.95(1.46-2.72)$ & $0.037^{*}$ \\
\hline LDL-C (mg/dl) & $98 \pm 29$ & $99 \pm 36$ & $98 \pm 28$ & $96 \pm 30$ & NS \\
\hline $\mathrm{HDL}-\mathrm{C}(\mathrm{mg} / \mathrm{dl})$ & $56 \pm 13$ & $58 \pm 13$ & $56 \pm 13$ & $58 \pm 13$ & NS \\
\hline Triglyceride (mg/dl) & $73 \pm 40$ & $72 \pm 48$ & $72 \pm 44$ & $67 \pm 43$ & NS \\
\hline Uric acid (mg/dl) & $4.3 \pm 1.0$ & $4.4 \pm 1.0$ & $4.4 \pm 0.9$ & $4.3 \pm 1.0$ & NS \\
\hline $\mathrm{CRP}(\mathrm{mg} / \mathrm{dl})$ & $0.13(0.06-0.34)$ & $0.12(0.06-0.29)$ & $0.11(0.06-0.22)$ & $0.10(0.06-0.24)$ & 0.20 \\
\hline GFR $\left(\mathrm{ml} / \mathrm{min} / 1.73 \mathrm{~m}^{2}\right)$ & $94 \pm 19$ & $93 \pm 18$ & $90 \pm 18$ & $92 \pm 18$ & NS \\
\hline
\end{tabular}

Data were expressed as Mean \pm SD or Median (Interquartile range). SBP indicates systolic blood pressure; BMI, body mass index; HOMA-IR, Homeostasis Model Assessment for insulin resistance; LDL-C, low density lipoproteins cholesterol; HDL-C, high density lipoproteins cholesterol; CRP, C-reactive protein; $A C R$, albumin creatinine ratio; GFR, glomerular filtration rate; mtDNA, mitochondrial DNA; NS, not significant. *Difference was statistical significant between quartile II and IV.

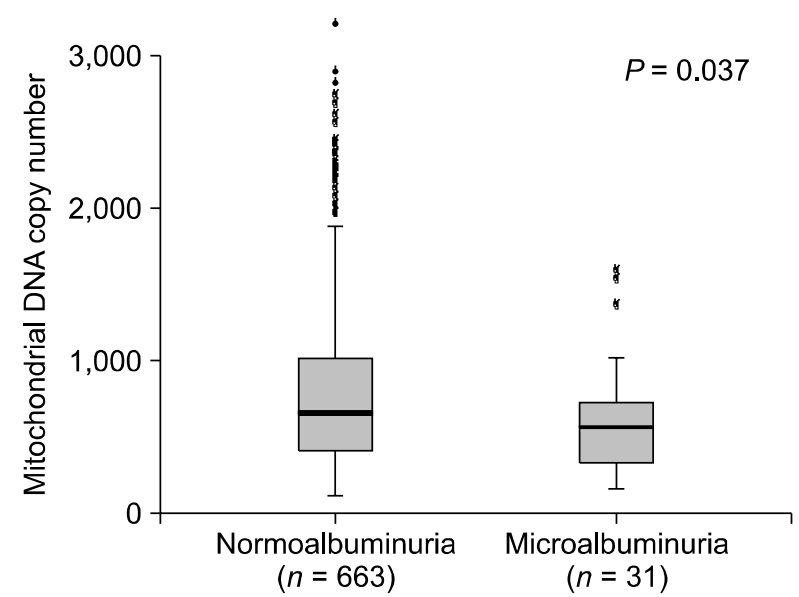

Figure 1. Distribution of mitochondrial DNA copy number by albuminuria status $(P=0.037)$.

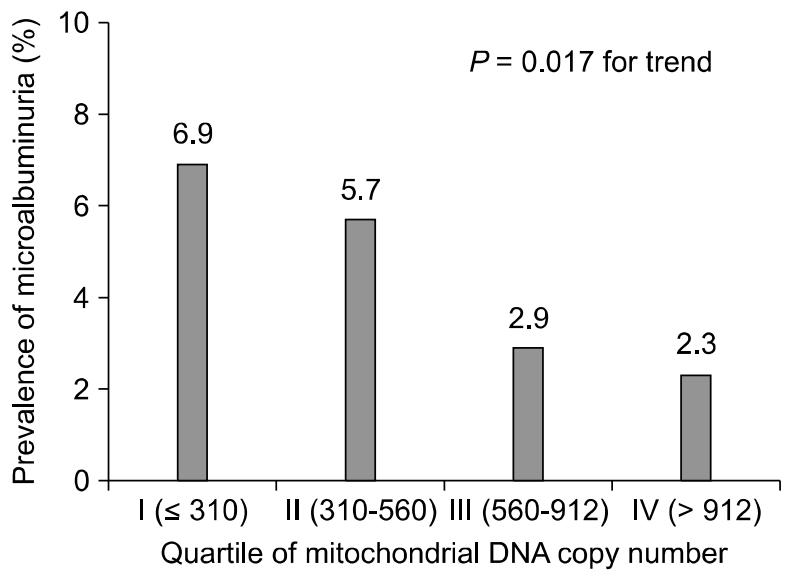

Figure 2. The prevalence of microalbuminuria according to the quartiles of mitochondrial DNA copy number $(P=0.012$ for trend). 
seline characteristics of the study population by quartile of mitochondrial DNA copy number. Subjects in the upper mtDNA quartiles were younger than those in the lower quartiles. SBP, fasting glucose, and BMI did not show any significant differences between the quartile groups divided by the number of the mtDNA copy. In addition, HOMA-IR scores did not show any consistent changes across the quatile groups, while the scores were different from each group tested by Kruskal-Wallis test.

We compared the median mtDNA copy number according to microalbuminuria status. Thirty-one subjects with microalbuminuria had a median mtDNA copy number of 467 (228-658), which was lower than median mtDNA copy number in subjects with normoalbuminuria $[565$ (312-928), $P=$ 0.037] (Figure 1).

Figure 2 presents the prevalence of microalbuminuria according to the quartiles of mtDNA copy number. The prevalence of microalbuminuria decreased progressively from the lower to the upper quartiles of mtDNA copy number $(6.9 \%$, $5.7 \%, 2.9 \%$, and $2.3 \%$ in quartiles $1,2,3$, and 4 , respectively, $P=0.017$ for trend).

Finally, the independent relationship between mtDNA copy number and microalbuminuria was evaluated by multiple logistic regression analyses, which adjusted for sex, age, smoking status, physical activity, SBP, DBP, BMI, waist circumference, HOMA-IR score, WBC counts, LDL cholesterol, HDL cholesterol, triglyceride, uric acid, CRP, and GFR (Table 2). The quartiles of mtDNA copy number were independently associated with the prevalence of microalbuminuria $(P=0.011$ for trend). Compared with the lowest quartile, the highest quartile had an odds ratio (OR) of 0.21 for microalbuminuria $[95 \%$ (confidence interval) $\mathrm{Cl}, 0.05$ to $0.83 ; P=0.026]$. Each 10-copy increments in mtDNA was associated with an OR of 0.988 for microalbuminuria $(95 \% \mathrm{Cl}, 0.977$ to $0.999 ; P=0.027)$.

Table 2. Risk for microalbuminuria of mitochondrial DNA copy number: multiple logistic regression analyses.

\begin{tabular}{lcl}
\hline $\begin{array}{c}\text { Mitochondrial DNA } \\
\text { copy number }\end{array}$ & Odds ratio $(95 \% \mathrm{Cl})$ & $P$ \\
\hline Quartile 2* $^{*}$ & $0.73(0.28-1.91)$ & $\mathrm{NS}$ \\
Quartile 3* $^{*}$ & $0.38(0.12-1.19)$ & 0.09 \\
Quartile 4* & $0.26(0.05-0.83)$ & 0.03 \\
per 10 copy increment & $0.988(0.977-0.999)$ & 0.03 \\
\hline
\end{tabular}

${ }^{*}$ Compared with quartile 1. These models were adjusted for sex, age, smoking status, physical activity, SBP, DBP, BMI, waist circumference, HOMA-IR score, WBC count, LDL-cholesterol, HDL-cholesterol, triglyceride, uric acid, CRP, and GFR. NS indicates not significant.

\section{Discussion}

We found a negative association between mtDNA copy number in peripheral blood and the prevalence of microalbuminuria in a community based population. Individuals in the higher quartile of mtDNA copy number had a lower prevalence of microalbuminuria. Previous studies have reported a correlation between mtDNA copy number and several cardiovascular risk factors such as aging and insulin resistance (Lee et al., 1998; Song et al., 2001). Our population-based study demonstrated that after adjusting for various cardiovascular risk factors, mtDNA copy number had an independent, negative association with microalbuminuria. Microalbuminuria reflects vascular damage and appears to be a surrogate marker of early atherosclerosis in patients with or without diabetes and/or hypertension (de Zeeuw, 2004). This negative association between mtDNA copy number and microalbuminuria suggests that low mtDNA content may be involved in the pathogenesis of endothelial dysfunction. It has become increasingly evident that decreased mitochondrial oxidative phosphorylation contributes to the initiation and development of atherosclerosis. Atherosclerotic patients show greater mtDNA damage in both the heart and the aorta than do healthy controls (Corral-Debrinski et al., 1992; Ballinger et al., 2002). Cardiovascular disease risk factors such as tobacco smoke exposure and hypercholesterolemia are associated with increased mtDNA damage (KnightLozano et al., 2002).

Reactive oxygen species (ROS) may be one pathway linking mitochondrial dysfunction with atherosclerosis, because mitochondrial respiratory chain is a major source of ROS as byproducts of normal cell respiration. Mitochondrial dysfunction may impair respiratory chain function and thereby increase the electron leak in the electron transport chain and ROS production (Puddu et al., 2005). mtDNA is also vulnerable to damage by ROS, because it is not protected by histone proteins and is located closed to the inner mitochondrial membrane, where ROS are generated (Croteau et al., 1999). Increased production of ROS in mitochondria damages mtDNA and decreases the steady-state level of mtDNA-encoded mRNA transcripts and mitochondrial protein synthesis in vascular smooth muscle cells (Ballinger et al., 2000). This may generate a vicious cycle leading to progressive accumulation of mtDNA damage and ROS production, and, consequently, atherosclerosis (Heinecke, 1998).

It has not been demonstrated whether reduced mtDNA copy number in peripheral blood reflects decreased capacity for oxidative phosphorylation. 
However, mtDNA copy number in peripheral blood correlates with insulin resistance in the offspring of type 2 diabetic patients (Song et al., 2001; Cho et al., 2007). Impaired mitochondrial function measured directly by nuclear magnetic resonance is associated with insulin resistance in the elderly (Petersen et al., 2003). The rates of mitochondrial phosphorylation in skeletal muscle is lower in insulin-resistant individuals than in healthy control (Petersen et al., 2004). Moreover, expression of nuclear-encoded genes involved in oxidative phosphorylation is down-regulated in insulin-resistant skeletal muscle (Mootha et al., 2003). From these similar observations, we speculate that mtDNA copy number in peripheral blood cells is a surrogate marker of whole body mitochondrial function.

Mitochondrial dysfunction and overproduction of ROS have emerged as a potential mechanism of renal complication in diabetes and hypertension (Nishikawa et al., 2000a, b; de Cavanagh et al., 2006). Proteinuria and mitochondrial ROS production simultaneously higher in the hypertensive rat than in normotensive rats (de Cavanagh et al., 2006). In addition, gene delivery to reduce mitochondrial ROS production attenuates proteinuria and renal hypertrophy in diabetic mice (Zhang et al., 2006). It is still unclear whether mitochondrial ROS production is a common pathway to induce proteinuria and renal progression. Conversely, albumin exposure activates mitochondrial apoptotic pathways and increases ROS production in proximal tubule cells (Erkan et al., 2007).

Our study has some limitations. Because it was a cross-sectional study, we could not examine the effect of reduced mtDNA content over time. Only one morning urine sample was obtained to measure ACR. This is commonly done in epidemiologic studies, but in the clinical setting, it is recommended that several samples should be obtained for each subject because of microalbuminuria variability and false-positive results (Molitch et al., 2003). Our sample was exclusively Asian, which may limit generalizations from our findings. Our population had several unique characteristics; such as its geographic isolation and social structure comprising large extended families, which maintain a nomadic lifestyle and have little access to health services. Our population was much younger and had a lower prevalence of hypertension, diabetes, dyslipidaemia, and obesity than other population-based studies. These characteristics can explain the low prevalence of microalbuminuria in our study.

In conclusion, higher mtDNA copy number was independently associated with lower prevalence of microalbuminuria in our community-based study.
This suggests that reduced mtDNA content in peripheral blood may be involved in the pathogenesis of endothelial dysfunction. Further study is needed to clarify whether mtDNA content of peripheral blood is associated with mitochondrial function in the steady state.

\section{Methods}

\section{Study population}

Existing DNA samples and data from the GENe DIScovery for Complex traits in Asian population of Northeast (GENDISCAN) project were used in this study. The GENDISCAN project was designed originally to investigate genetic susceptibility to disease with complex trait disease and was conducted in Dashbalbar, Dornot Province, Mongolia, from 2005 to 2006 (Gombojav et al., 2008). Investigators requested that all inhabitants older than 10 years participate in this cross-sectional survey; 2327 subjects (about 58\% all inhabitants older than 10 years) responded to the investigators' request, from which the investigators of the GENDISCAN project selected 1002 individuals as predefined criteria. The participants underwent a medical interview and physical examination and provided a fasting blood sampling for DNA analysis and other laboratory tests, and a random urine sample. The institutional review board (IRB) of Ministry of Health and Welfare in Mongolia and the IRB of Seoul National University in Korea approved the GENDISCAN project, and all participants gave written informed consents.

For this study, we excluded 283 children (younger than 18 years) and 25 patients with chronic kidney disease (CKD), which left 694 subjects for the primary analysis.

\section{Assessment of clinical parameters}

Data about physical activity and smoking were recorded. Smoking status was categorized as smoker (meaning a person who currently smoked or one who had smoked within one year) and non-smoker. A physically active person was defined as someone who exercised or performed physical work for more than 30 min three times a week. Blood pressure was measured in the sitting position after a five-minute rest. And the averages of three measurements were recorded. Hypertension was defined as systolic blood pressure (SBP) of at least $140 \mathrm{mmHg}$ or diastolic blood pressure (DBP) of at least $90 \mathrm{mmHg}$. Body mass index (BMI) was computed as weight in kilograms divided by the square of height in meters. The waist circumference at the umbilicus was measured while the subject stood and was rounded to the nearest centimeter. An eight hour fasting blood sample was taken to determine the concentrations of triglyceride, LDL cholesterol, HDL cholesterol, glucose, uric acid, insulin, and C-reactive protein (CRP). Diabetes was defined as a fasting blood glucose level of $126 \mathrm{mg} / \mathrm{dl}$ or more. Homeostasis Model Assessment for insulin resistance (HOMA-IR) score was calculated by the following formula: [fasting serum insulin $(\mu \mathrm{U} / \mathrm{ml}) \times$ fasting plasma glucose $(\mathrm{mg} / \mathrm{dl})] / 405$. Glomerular filtration rate (GFR) was estimated by the abbreviated 
MDRD Study Equation (Levey et al., 1999). A random urine sample was obtained to determine the albumin to creatinine ratio (ACR). Urinary albumin concentration was measured by an immunoturbidimetric assay and urinary creatinine concentration was measured by the Jaffe rate reaction. Microalbuminuria was defined as an ACR of 30 to $299 \mathrm{mg} / \mathrm{g}$ for both men and women. Subjects with an ACR of $<30 \mathrm{mg} / \mathrm{g}$ were defined as having normoalbuminuria. CKD was defined as a reduced estimated GFR $(<60$ $\mathrm{ml} / \mathrm{min} / 1.73 \mathrm{~m}^{2}$ ) or macroalbuminuria (ACR $\geq 300 \mathrm{mg} / \mathrm{g}$ ). Patients with CKD were excluded from the study.

\section{Quantitation of mtDNA copy number}

We purified the total cellular DNA from peripheral leukocytes, and measured the mtDNA copy number and nuclear DNA copy number using the real time PCR method with an ABI Prism 7900HT (Applied Biosystems) as described previously (Song et al., 2001). The mtDNA copy number was corrected by $28 \mathrm{~S}$ rRNA contents. We used the specific fluorescent probes for mtDNA and 28S rRNA. They were labeled internally using the fluorescent dyes 5-carboxyfluorescein (FAM) on the 5' end, and 6-carboxy-tetramethylrhodamine (TAMRA) on the $3^{\prime}$ end. We used the primers for mtDNA, mt2981 (5'ACGACCTCGATGTTGAGATC3') and $m \mathrm{t} 3245$ (5'GCTCTGCCATCTTAACAAACC3'), with the mtDNA probe together (5'FAM-TTCAGACCGGAGTAATCCAGGTCG-TAMRA3' made to nt 3071-3095). The primers of 28S rRNA were 28S-7358 (5'TTAAGGTAGCCAAAAATGCCTCG3') and 28S-7460 (5'CCTTGGCTGTGGTTTCGCT3'). The 28S rRNA probe was 5'-FAM-TGAACGAGATTCCCACTGTCCCCCTACCTACTAAATCTAMRA3' made to nt7408-7440.

We added DNA to the PCR mixture which contained the primers (10 pmol each), $200 \mathrm{nM}$ Taqman probe, Premix Ex Taq $200 \mathrm{nM}$ (Takara bio Inc) and performed the PCR amplification for mtDNA and the reference 28S rRNA separately. We measured the amount of target by a standard curve of $\mathrm{Ct}$ values from known concentrations of the standard plasmid DNA with mtDNA and 28S rRNA, respectively. The following conditions was used for amplification; one cycle of $50^{\circ} \mathrm{C}$ for $2 \mathrm{~min}$, one cycle of $95^{\circ} \mathrm{C}$ for $10 \mathrm{~min}$, and 40 cycles of $95^{\circ} \mathrm{C}$ for $15 \mathrm{~s}$ and $60^{\circ} \mathrm{C}$ for $1 \mathrm{~min}$.

\section{Statistical analysis}

Baseline characteristics were assessed according to quartile of mtDNA copy number. Difference between the quartile groups were tested for statistical significance by $\chi^{2}$ test for categorical variables and one way ANOVA for continuous variables except for CRP levels and HOMA-IR score. CRP levels and HOMA-IR score were compared using the Kruskal-Wallis test. mtDNA copy number was compared between normoalbuminuria group and microalbuminuria group using the Mann-Whitney test. To assess the independent effect of mtDNA copy number on microalbuminuria, we used multiple logistic regression analyses adjusted for sex, age, smoking status, physical activity, SBP, DBP, BMI, waist circumference, HOMA-IR score, white blood cell (WBC) counts, LDL cholesterol, HDL cholesterol, triglyceride, uric acid, CRP, and GFR. CRP levels were highly skewed and were normalized by logarithmic transformation in regression analyses. A $P$-value of less than 0.05 was considered significant. All the statistical analyses for this study were conducted with SPSS 13.0 for Windows.

\section{Acknowledgements}

We would like to thank all the individuals and their families for participation in this study. Korean government approved and has supported the pilot study, And phase I study (Ministry of Education Science and Technology, Grant \#M10305030000). We sincerely thank Young Min Cho for a critical reading of the manuscript.

\section{References}

Antonetti DA, Reynet C, Kahn CR. Increased expression of mitochondrial-encoded genes in skeletal muscle of humans with diabetes mellitus. J Clin Invest 1995;95:1383-8

Ballinger SW, Patterson C, Yan CN, Doan R, Burow DL, Young CG, Yakes FM, Van Houten B, Ballinger CA, Freeman $B A$, Runge MS. Hydrogen peroxide- and peroxynitriteinduced mitochondrial DNA damage and dysfunction in vascular endothelial and smooth muscle cells. Circ Res 2000;86:960-6

Ballinger SW, Patterson C, Knight-Lozano CA, Burow DL, Conklin CA, Hu Z, Reuf J, Horaist C, Lebovitz R, Hunter GC, Mclntyre K, Runge MS. Mitochondrial integrity and function in atherogenesis. Circulation 2002;106:544-9

Cho YM, Park KS, Lee HK. Genetic factors related to mitochondrial function and risk of diabetes mellitus. Diabetes Res Clin Pract 2007;77 Suppl 1:S172-7

Corral-Debrinski M, Shoffner JM, Lott MT, Wallace DC. Association of mitochondrial DNA damage with aging and coronary atherosclerotic heart disease. Mutat Res 1992; 275:169-80

Croteau DL, Stierum RH, Bohr VA. Mitochondrial DNA repair pathways. Mutat Res 1999;434:137-48

de Cavanagh EM, Toblli JE, Ferder L, Piotrkowski B, Stella I, Inserra F. Renal mitochondrial dysfunction in spontaneously hypertensive rats is attenuated by losartan but not by amlodipine. Am J Physiol Regul Integr Comp Physiol 2006;290:R1616-25

de Zeeuw D. Albuminuria, not only a cardiovascular/renal risk marker, but also a target for treatment. Kidney Int Suppl 2004:S2-6

Erkan E, Devarajan P, Schwartz GJ. Mitochondria are the major targets in albumin-induced apoptosis in proximal tubule cells. J Am Soc Nephrol 2007;18:1199-208

Gombojav B, Park H, Kim JI, Ju YS, Sung J, Cho SI, Lee MK, Ohrr H, Radnaabazar J, Seo JS. Heritability and linkage study on heart rate in a Mongolian population. Exp Mol Med 2008;40:558-64

Heinecke JW. Oxidants and antioxidants in the pathogenesis of atherosclerosis: implications for the oxidized low density lipoprotein hypothesis. Atherosclerosis 1998;141:1-15 
Knight-Lozano CA, Young CG, Burow DL, Hu ZY, Uyeminami $D$, Pinkerton KE, Ischiropoulos H, Ballinger SW. Cigarette smoke exposure and hypercholesterolemia increase mitochondrial damage in cardiovascular tissues. Circulation 2002;105:849-54

Lee HK, Song JH, Shin CS, Park DJ, Park KS, Lee KU, Koh CS. Decreased mitochondrial DNA content in peripheral blood precedes the development of non-insulin-dependent diabetes mellitus. Diabetes Res Clin Pract 1998;42:161-7

Levey AS, Bosch JP, Lewis JB, Greene T, Rogers N, Roth D. A more accurate method to estimate glomerular filtration rate from serum creatinine: a new prediction equation. Modification of Diet in Renal Disease Study Group. Ann Intern Med 1999;130:461-70

Lowell BB, Shulman GI. Mitochondrial dysfunction and type 2 diabetes. Science 2005;307:384-7

Madamanchi NR, Runge MS. Mitochondrial dysfunction in atherosclerosis. Circ Res 2007;100:460-73

Molitch ME, DeFronzo RA, Franz MJ, Keane WF, Mogensen $\mathrm{CE}$, Parving $\mathrm{HH}$. Diabetic nephropathy. Diabetes Care 2003;26 Suppl 1:S94-8

Mootha VK, Lindgren CM, Eriksson KF, Subramanian A, Sihag S, Lehar J, Puigserver P, Carlsson E, Ridderstrale M, Laurila E, Houstis N, Daly MJ, Patterson N, Mesirov JP, Golub TR, Tamayo P, Spiegelman B, Lander ES, Hirschhorn JN, Altshuler D, Groop LC. PGC-1alpha-responsive genes involved in oxidative phosphorylation are coordinately downregulated in human diabetes. Nat Genet 2003;34: 267-73

Nishikawa T, Edelstein D, Brownlee M. The missing link: a single unifying mechanism for diabetic complications. Kidney Int Suppl 2000a;77:S26-30
Nishikawa T, Edelstein D, Du XL, Yamagishi S, Matsumura T, Kaneda Y, Yorek MA, Beebe D, Oates PJ, Hammes HP, Giardino I, Brownlee M. Normalizing mitochondrial superoxide production blocks three pathways of hyperglycaemic damage. Nature 2000b;404:787-90

Nisoli E, Clementi E, Carruba MO, Moncada S. Defective mitochondrial biogenesis: a hallmark of the high cardiovascular risk in the metabolic syndrome. Circ Res 2007;100: 795-806

Petersen KF, Befroy D, Dufour S, Dziura J, Ariyan C, Rothman DL, DiPietro L, Cline GW, Shulman GI. Mitochondrial dysfunction in the elderly: possible role in insulin resistance. Science 2003;300:1140-2

Petersen KF, Dufour S, Befroy D, Garcia R, Shulman GI. Impaired mitochondrial activity in the insulin-resistant offspring of patients with type 2 diabetes. N Engl J Med 2004; 350:664-71

Puddu P, Puddu GM, Galletti L, Cravero E, Muscari A. Mitochondrial dysfunction as an initiating event in atherogenesis: a plausible hypothesis. Cardiology 2005;103: $137-41$

Song J, Oh JY, Sung YA, Pak YK, Park KS, Lee HK. Peripheral blood mitochondrial DNA content is related to insulin sensitivity in offspring of type 2 diabetic patients. Diabetes Care 2001;24:865-9

Zhang Y, Wada J, Hashimoto I, Eguchi J, Yasuhara A, Kanwar YS, Shikata K, Makino H. Therapeutic approach for diabetic nephropathy using gene delivery of translocase of inner mitochondrial membrane 44 by reducing mitochondrial superoxide production. J Am Soc Nephrol 2006;17:1090101 